\title{
Foundations to frontiers of big data analytics
}

\begin{abstract}
In recent times, big data analytics has become a major trend in catering data queries that has been growing dramatically. The present paper gives a brief description of latest happenings of Big Data analytics. A case study using Spark is given as an example. The paper gives the importance of cloud computing in Big data paradigm.
\end{abstract}

Keyword: Big data; Map reduce; Spark; Cloud computing; Hadoop; Haloop 